\title{
Update on treatment of melioidosis
}

\author{
W Chierakul
}

Melioidosis, a Gram negative bacterial disease, is endemic in Southeast Asia and Northern Australia. Clinical manifestations of the disease vary from mild localized disease to severe fatal septicaemia. The mortality rate remains high and may reach $30 \%$ in some areas. Treatment is divided into two phases; acute intensive phase and oral eradication phase. Acute intensive phase starts with intravenous antibiotics.

Ceftazidime, one of the third generation cephalosporins, remains the gold standard treatment, especially in Thailand. Meropenem is used in patients with severe septicaemia in Australia. Intravenous injection continues for at least 2 weeks or until clear clinical responses occur, such as defervescence, gaining appetite, improved general well-being, etc. Neurological and rheumatological involvements always need longer intensive treatment, thus combination therapy in these groups are controversial.

Trimethoprim-sulphamethoxazole for 12-20 weeks remains the first line treatment for oral eradication phase. Patients who are hypersensitive to sulfa should use amoxicillin-clavulanate with at least 3 times daily dosing interval. The regimen is inferior to the first line treatment.

Department of Clinical Tropical Medicine, Faculty of Tropical Medicine, Mahidol University, Thailand Address for correspondence: Dr Wirongrong Chierakul, Department of Clinical Tropical Medicine, Faculty of

Tropical Medicine, Mahidol University, Thailand +662 3549100. Email: wirongrong.chi@mahidol.ac.th

(D) https://orcid.org/0000-0003-2103-9425 